health clinic, with specific staffing allocation, to ensure a more thorough and efficient way to address physical health.

\section{Diabetes and liaison psychiatry: the characteristics of patients with diabetes referred to a liaison psychiatry service in London}

\author{
Alexandra Simpson ${ }^{1 *}$, Lucy Bradford ${ }^{2}$ and Marilia Calcia ${ }^{2}$ \\ ${ }^{1}$ East Sussex Healthcare NHS Trust and ${ }^{2}$ King's College London \\ ${ }^{*}$ Corresponding author.
}

doi: 10.1192/bjo.2021.309

Aims. To determine the characteristics of adult patients referred to a Liaison Psychiatry service in a general teaching hospital in London, UK with 950 inpatient adult beds.

Method. All referrals for adult inpatient psychiatric consultation made during a period of 9 months were reviewed; those that involved a patient with a diagnosis of diabetes were analysed. Descriptive statistics were used; data were collected on demographic characteristics and physical and mental health parameters, including type of diabetes, number of years since diabetes diagnosis, glycaemic control, presence of diabetes-related complications, reason for Psychiatry consultation request, psychiatric diagnosis, psychotropic medication, frequency of admissions to general hospital, psychiatric risk issues and outcome of psychiatric consultation.

Result. Pilot results indicate that 30 diabetic patients were referred for a psychiatric consultation in 9 months. Of those, 9 had type 1 diabetes, 17 had type 2 diabetes and 1 had pre-diabetes 3 were unknown. 13 were male and 17 were female; the median age was 46 (range 18 to 68); the ethnicities were 6 White, 15 Black, 1 Asian and 8 other.

Diabetes-related complications were present in $77 \%$ (retinopathy $10 \%$, kidney disease $27 \%$, neuropathy $13 \%$, diabetic foot $16 \%$ ). $6 \%$ had comorbid cardiovascular disease. $10 \%$ were on dialysis and 3\% had had amputations.

The main reason for referral for psychiatric consultation was low mood and self harm; other reasons were recurrent DKA, anxiety and self neglect. Psychiatric risk issues included 20\% risk of self-harm/suicide; $13 \%$ risk of violence; 10 risk of selfneglect. The outcomes of liaison psychiatry consultation were: $30 \%$ received an assessment that led to recommendations to the general medical team and did not require further psychiatric input; $27 \%$ received continued psychiatric follow-up during the admission. With regards to treatment, 36\% had psychiatric treatment (including medication) reviewed; 47\% received general treatment recommendations, including recommendations for new laboratory or radiological investigations or change in level of nursing care. $20 \%$ required transfer to an inpatient psychiatric unit, with $33 \%$ discharged to care of community mental health.

Conclusion. Our findings indicate the scope of practice for a Liaison Psychiatry service with regards to adult hospital inpatients with diabetes. Our data suggest that patients with type 2 diabetes are the majority of inpatients with diabetes that require psychiatric consultations, and that the majority of those are patients already known to psychiatric services due to long-term severe mental disorders, particularly schizophrenia, schizoaffective disorder or bipolar disorder. Most of those patients have medical comorbidities and severe diabetes-related complications. Patients with type 1 diabetes, despite making up a smaller proportion of referrals for psychiatric consultations, also tend to have recurrent hospital admissions and features of self-neglect.
Implementation of physical examination pro forma - a complete audit cycle

Deshwinder Singh Sidhu ${ }^{1 *}$ and Guy Molyneux ${ }^{2}$

${ }^{1}$ Phoenix Care Centre and ${ }^{2}$ HSE St. Aloysius Mater Misericordiae University Hospital

${ }^{\star}$ Corresponding author.

doi: 10.1192/bjo.2021.310

Aims. Aim of this audit is to achieve and maintain 100\% compliance in physical examination on admission.

Background. Conducting physical examination on admission is a mandatory requirement and is monitored by the Mental Health Commission during yearly inspections. A report published by Inspectorate of the Mental Health Commission recently in 2019 identifies a gap in physical health monitoring. We conducted a complete audit cycle in an inner city hospital psychiatric ward to monitor compliance with physical examination on admission. Method. We based the audit on Judgment Support Framework (JSF) version 5 standards. A retrospective review of all of the patient's medical records was carried out. 13 medical records were reviewed in the first cycle. The results of the first cycle were presented to the Multi Disciplinary Team (MDT) members, including the Non-Consultant Hospital Doctors (NCHD). Physical health policy was reviewed, in consultation with the committee and Clinical Director, a Physical Examination pro-forma (colour coded) was developed and implemented. It was based on the National Guidelines and the JSF ver.5. All members of the MDT and NCHDs were briefed on the pro forma introduced. A repeat audit cycle was conducted of all patients admitted after first audit cycle. Data were collected using a simple audit tool indicating if physical examination was conducted or refused.

Result. A total of 22 medical records were audited. 13 medical records in the first cycle indicted only 3 patients had physical examination on admission. However, prior to admission a total of six patients had physical exam in the Emergency Department (ED). Upon implementation of the pro forma, 9 medical records of all patients admitted post-first cycle were audited. A total of 7 patients had physical examination on admission to the ward. Two patients refused physical examination and this was clearly documented. One patient had physical examination completed in ED. All newly admitted patients had physical examination completed or the reason why it wasn't completed documented clearly. Conclusion. Physical examination pro forma was successfully implemented, raising current compliance to a $100 \%$, with a significant improvement from $23 \%$ compliance in the first cycle. Existing pro forma is helpful as a reminder to NCHDs. Colour coding of pro forma improves accessibility and distinguishability during the process of admission and auditing. Physical examination pro forma will be audited every 6 monthly.

\section{Audit on monitoring physical health of patients on mood stabilisers following NICE guidelines}

Sathyan Soundararajan*, Asha Dhandapani and Claire Jones BCUHB

${ }^{\star}$ Corresponding author.

doi: 10.1192/bjo.2021.311

Aims. The aim is to find out if the physical health monitoring is adhered to in accordance with NICE guidelines in individuals with Intellectual disability who are on mood stabilisers and known to LD services. 